$$
\begin{gathered}
\text { CERN-SL 93-14 AP } \\
\operatorname{sug} 918 \quad c_{1}
\end{gathered}
$$

EUROPEAN LABORATORY FOR PARTICLE PHYSICS

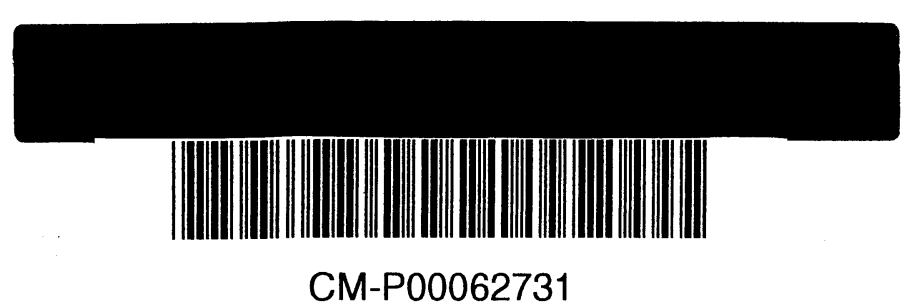

DAPNIA/SEA/93-01

CERN SL/AP 93-14

\title{
A Generalized Method for Calculating Wake Potentials
}

\author{
Olivier Napoly * \\ Yong Ho Chin ${ }^{\dagger}$ \\ Bruno Zotter
}

\begin{abstract}
We describe a generalized method to compute wake potentials created in axisymmetric structuces. It relies on expressing the wake potentials, of any multipole order, as integrals over the e.m. fields along an arbitrary one-dimensional contour spanning the structure longitudinally. For perfectly conducting structures, the integration along the axis can then be replaced by choosing a contour beginning and ending on the beam tubes. Thus it generalizes the former method of calculating the wake potentials by integrating along a straight line at the beam tube radius. Its usefulness is illustrated with the computer code $\mathrm{ABCI}$ which permits calculation of wake potentials in structures extending to the inside of the beam tube radius, or having unequal beam tube radii at the two sides.
\end{abstract}

Geneva, Switzerland

19 March 1993

*DAPNIA-SEA, CE Saclay

${ }^{\dagger}$ LBL Berkeley 


\section{Introduction}

The determination of the wake potentials and impedances created by (highly conducting) metallic structures surrounding the beam trajectory is an important issue in the design of accelerators. In most practical cases, the wake fields must be calculated with computer codes. For structures which are symmetric about the beam axis, a multipolar Fourier expansion of the potentials with respect to the azimuthal angle $\theta$ can be used and reduces the problem to a 2d-calculation. Moreover, for cavity-like structures, using the known radial dependence of the monopolar $(m=0)$ longitudinal and dipolar $(m=1)$ transverse potentials, the integration of the wake fields can be performed along a straight line parallel to the axis at the beam tube radius [1].

For perfectly conducting walls, the boundary conditions ensure that the integral along the beam tube vanishes for the tangential (longitudinal or azimuthal) components of the wake field. This leaves the integral across the cavity gap as the only contribution to the wake potentials. Such a simplification is essential for computer calculations, in particular of large structures and for short bunches requiring small mesh size, where long beam tubes would require excessive computer memory and cpu time. However, this technique works only if the two beam tubes have equal radii, and when no part of the cavity extends to a smaller radius. If this is not the case, for instance for single tapers and steps, collimators, or cavities with small aperture irises, the only alternative is to integrate along a straight line at an allowable radius, and with beam tubes as long as possible. Usually it is then also necessary to subtract the wake potential of the beam tubes without structure ("numerical noise") which is different from zero due to the discretization of the geometry.

It has been realized already some time ago $[2,3]$ that the longitudinal $(m=0)$ and transverse (azimuthal) $(m=1)$ potentials can also be calculated by integrating the wake fields along the (perfectly conducting) boundary of a structure. The contribution of the beam tubes still vanishes, and one can treat more general structures without having to introduce long beam tubes. The only limitation of this method is for the transverse wake potential when the two beam tubes have unequal radii.

In this paper, we further generalize the above result by showing that the longitudinal and transverse wake potentials, at all orders $m$ in the multipolar expansion, can be expressed as integrals over the wake fields along any arbitrary contour spanning the structure longitudinally. Except for $m \geq 1$ and unequal beam tube radii, the integrand can be chosen such that the contribution of the (perfectly conducting) beam tubes vanishes. After having introduced our notations in Section 2, we derive this result in Section 3. We discuss the implementation of the method in the computer code ABCI [4] in Section 4. For convenience, the contours used in the code are composed of three longitudinal line segments at different radii connected by two radial segments. In this way, one can adapt the contour to the beam tube radii, while the inner segment stretches below the aperture of the structure. The examples of a collimator, of sudden steps (in, out, or double) in beam tubes, and of a constant-gradient accelerating structure for a linear collider, all results obtained with ABCI, are shown in the figures. 


\section{Definitions and Notations}

The longitudinal and transverse wake potentials are defined by

$$
W_{z}(r, \theta, s) \stackrel{\text { def }}{=}-\frac{1}{Q} \int_{-\infty}^{+\infty} d z E_{z}(r, \theta, z, t(z, s))
$$

and

$$
\mathbf{W}_{\perp}(r, \theta, s) \stackrel{\text { def }}{=} \frac{1}{Q} \int_{-\infty}^{+\infty} d z\left(\mathbf{E}_{\perp}+\mathbf{v} \times \mathbf{B}\right)(r, \theta, z, t(z, s))
$$

where $s$ is the distance behind a given origin $z_{0}=v t$ in the exciting bunch, and

$$
t(z, s)=(z+s) / v
$$

We assume that the bunch which excites wake fields in the structure has velocity $v=c$, total charge $Q$ and is characterized by a longitudinal charge distribution $\lambda(s)$ normalized to one, i.e.

$$
\int_{-\infty}^{+\infty} \lambda(s) d s=1
$$

It is convenient to decompose the electromagnetic fields as follows

$$
\begin{aligned}
& \mathbf{E}=\mathbf{E}^{(0)}+\mathbf{E}^{(r)} \\
& \mathbf{B}=\mathbf{B}^{(0)}+\mathbf{B}^{(r)}
\end{aligned}
$$

where $\left(\mathbf{E}^{(0)}, \mathbf{B}^{(0)}\right)$ are the e.m. fields which would be generated by the charge distribution $Q \lambda(s)$ in free space. The additional fields $\left(\mathbf{E}^{(r)}, \mathbf{B}^{(r)}\right)$, sometimes called 'radiated fields', are therefore solutions of the homogeneous Maxwell equations. The wake potentials $W_{z}$ and $\mathbf{W}_{\perp}$ are entirely given by the latter fields.

Then, given $\mathbf{r}_{0}=\left(r_{0}, \theta_{0}=0\right)$ the transverse coordinates of the exciting bunch, the e.m. fields obey the following Fourier expansion

$$
\begin{aligned}
& \left(E_{r}, B_{\theta}, E_{z}\right)(r, \theta, z, t)=\sum_{m=0}^{\infty}\left(e_{r}, b_{\theta}, e_{z}\right)^{(m)}(r, z, t) \cos (m \theta) \\
& \left(B_{r}, E_{\theta}, B_{z}\right)(r, \theta, z, t)=\sum_{m=1}^{\infty}\left(b_{r}, e_{\theta}, b_{z}\right)^{(m)}(r, z, t) \sin (m \theta)
\end{aligned}
$$

The even or odd parity of the fields with respect to $\theta$ is a consequence of the symmetry with respect to the plane containing the axis and the exciting distribution at $\theta_{0}=0$. It is therefore also a property of the fields $\left(\mathbf{E}^{(0)}, \mathbf{B}^{(0)}\right)$ in free space, and by linearity of the radiated fields $\left(\mathbf{E}^{(r)}, \mathbf{B}^{(r)}\right)$.

In order to simplify our notations in the next section, we introduce for a generic field $\phi$ the following definition

$$
\bar{\phi}(z, s) \stackrel{\text { def }}{=} \phi(z, t(z, s))
$$

in such a way that

$$
\partial_{z} \bar{\phi}(z, s)=\left(\partial_{z}+\partial_{c t}\right) \phi(z, t(z, s))
$$


For instance, by combining the two Maxwell equations

$$
\begin{aligned}
\left(\nabla \times \mathbf{E}^{(r)}\right)_{\theta} & =\partial_{z} E_{r}^{(r)}-\partial_{r} E_{z}^{(r)}=-\partial_{t} B_{\theta}^{(r)} \\
c\left(\nabla \times \mathbf{B}^{(r)}\right)_{r} & =c\left(\frac{1}{r} \partial_{\theta} B_{z}^{(r)}-\partial_{z} B_{\theta}^{(r)}\right)=\partial_{c t} E_{r}^{(r)}
\end{aligned}
$$

one can easily obtain the following relation

$$
\partial_{r} \bar{E}_{z}^{(r)}-\partial_{z}\left(\bar{E}_{r}^{(r)}+c \bar{B}_{\theta}^{(r)}\right)=-\frac{1}{r} \partial_{\theta}\left(c \bar{B}_{z}^{(r)}\right)
$$

In the same way, combining the two equations

$$
\begin{aligned}
\left(\nabla \times \mathbf{E}^{(r)}\right)_{r} & =\frac{1}{r} \partial_{\theta} E_{z}^{(r)}-\partial_{z} E_{\theta}^{(r)}=-\partial_{t} B_{r}^{(r)} \\
c\left(\nabla \times \mathbf{B}^{(r)}\right)_{\theta} & =c\left(\partial_{z} B_{r}^{(r)}-\partial_{r} B_{z}^{(r)}\right)=\partial_{c t} E_{\theta}^{(r)}
\end{aligned}
$$

one gets

$$
\partial_{r}\left(c \bar{B}_{z}^{(r)}\right)+\partial_{z}\left(\bar{E}_{\theta}^{(r)}-c \bar{B}_{r}^{(r)}\right)=\frac{1}{r} \partial_{\theta} \bar{E}_{z}^{(r)}
$$

Equations (10) and (12) are at the basis of the derivation described below.

\section{Calculation of the wake potentials}

In this section, we derive the main result of the paper, namely the expression of the longitudinal potential $W_{z}^{(m)}(r, s)$, at each order $m$, in terms of an integral of the wake fields along any given contour across the structure. The case $m=0$ is treated separately because it is simpler and because the integration along perfectly conducting beam tubes can always be eliminated. For $m \geq 1$, the latter property holds only when the beam tube radii are equal on both sides of the structure. Finally, the transverse potential is derived from the longitudinal one by applying the Panofsky-Wenzel theorem [5].

\subsection{The longitudinal potential}

When the multipole expansion of the radiated fields $\left(\mathbf{E}^{(r)}, \mathbf{B}^{(r)}\right)$, like in Eq.(6), is taken into account, Eqs.(10) and (12) yield at each order $m$

$$
\begin{aligned}
{\left[\partial_{r} \bar{e}_{z}^{(r)}-\partial_{z}\left(\bar{e}_{r}^{(r)}+c \bar{b}_{\theta}^{(r)}\right)\right]^{(m)} } & =-\frac{m}{r}\left[c \bar{b}_{z}^{(r)}\right]^{(m)} \\
{\left[\partial_{r}\left(c \bar{b}_{z}^{(r)}\right)+\partial_{z}\left(\bar{e}_{\theta}^{(r)}-c \bar{b}_{r}^{(r)}\right)\right]^{(m)} } & =-\frac{m}{r}\left[\bar{e}_{z}^{(r)}\right]^{(m)}
\end{aligned}
$$

The sum and difference of the preceding two equations, can be written as

$$
\begin{aligned}
& \partial_{r}\left(r^{m}\left[\bar{e}_{z}^{(r)}+c \bar{b}_{z}^{(r)}\right]^{(m)}\right)=r^{m} \partial_{z}\left[\bar{e}_{r}^{(r)}+c \bar{b}_{\theta}^{(r)}-\bar{e}_{\theta}^{(r)}+c \bar{b}_{r}^{(r)}\right]^{(m)} \\
& \partial_{r}\left(r^{-m}\left[\bar{e}_{z}^{(r)}-c \bar{b}_{z}^{(r)}\right]^{(m)}\right)=r^{-m} \partial_{z}\left[\bar{e}_{r}^{(r)}+c \bar{b}_{\theta}^{(r)}+\bar{e}_{\theta}^{(r)}-c \bar{b}_{r}^{(r)}\right]^{(m)}
\end{aligned}
$$


Introducing the $2 \mathrm{~d}$-vectors $\overrightarrow{\mathrm{S}}^{(m)}$ and $\overrightarrow{\mathrm{D}}^{(m)}$ in the $(r, z)$-plane defined by

$$
\vec{S}^{(m)}=\left(\begin{array}{c}
r^{m}\left[\bar{e}_{r}^{(r)}+c \bar{b}_{\theta}^{(r)}-\bar{e}_{\theta}^{(r)}+c \bar{b}_{r}^{(r)}\right]^{(m)} \\
r^{m}\left[\bar{e}_{z}^{(r)}+c \bar{b}_{z}^{(r)}\right]^{(m)}
\end{array}\right)
$$

and

$$
\overrightarrow{\mathrm{D}}^{(m)}=\left(\begin{array}{c}
r^{-m}\left[\bar{e}_{r}^{(r)}+c \bar{b}_{\theta}^{(r)}+\bar{e}_{\theta}^{(r)}-c \bar{b}_{r}^{(r)}\right]^{(m)} \\
r^{-m}\left[\bar{e}_{z}^{(r)}-c \bar{b}_{z}^{(r)}\right]^{(m)}
\end{array}\right)
$$

Equations (14) express the fact that the one-forms defined by the vectors $\vec{S}^{(m)}$ and $\vec{D}^{(m)}$ are closed in the $(r, z)$-plane, i.e.

$$
\begin{aligned}
& \partial_{r} \mathrm{~S}_{z}^{(m)}(r, z, s)-\partial_{z} \mathrm{~S}_{r}^{(m)}(r, z, s)=0 \\
& \partial_{r} \mathrm{D}_{z}^{(m)}(r, z, s)-\partial_{z} \mathrm{D}_{r}^{(m)}(r, z, s)=0
\end{aligned}
$$

This implies that the vectors $\vec{S}^{(m)}$ and $\vec{D}^{(m)}$ derive from a potential and that their integral along a closed contour (enclosing the vacuum) vanishes. This property will allow us to deform the wake field integration along a straight line $\mathcal{L}_{r}$ at radius $r$ to any given contour $\mathcal{C}$ (cf. Fig.1).

\subsubsection{THE $m=0$ CASE}

The fields $(\mathbf{E}, \mathbf{B})^{(m=0)}$ and $\left(\mathbf{E}^{(0)}, \mathbf{B}^{(0)}\right)^{(m=0)}$ are the e.m. fields created by a hollow charge distribution of radius $r_{0}$ centered on the axis, moving inside the structure considered or in free space. It is well known that the free space solution, given by the non-zero field components

$$
\begin{aligned}
{\left[E_{r}^{(0)}\right]^{(m=0)}(r, z, t) } & =\frac{Q}{2 \pi \epsilon_{0} r} \lambda(c t-z) \\
c\left[B_{\theta}^{(0)}\right]^{(m=0)} & =\left[E_{r}^{(0)}\right]^{(m=0)}
\end{aligned}
$$

also describes the fields in a perfectly conducting beam tube. It therefore coincides with the asymptotic form of the fields $(\mathbf{E}, \mathbf{B})^{(m=0)}$ at both ends of the structure, i.e. for $z= \pm \infty$ and $s$ finite. As a consequence, one finds

$$
\left[\bar{E}_{z}^{(r)}, \bar{E}_{r}^{(r)}, \bar{B}_{\theta}^{(r)}\right]^{(m=0)}(r, z, s) \rightarrow 0 \quad \text { for } \quad z \rightarrow \pm \infty
$$

while the other components are zero. With the notations of Fig.1, one can deform the straight integration line $\mathcal{L}_{r}$ into any contour $\mathcal{C}$ across the structure, in such a way that

$$
\int_{\mathcal{L}_{r}} \overrightarrow{\mathrm{S}}^{(m=0)}(r, z, s) \cdot d \vec{l}=\int_{\mathcal{C}} \overrightarrow{\mathrm{S}}^{(m=0)}\left(r^{\prime}, z, s\right) \cdot d \vec{l}
$$

since, from Eq.(19), the integrals along the radial segments connecting $\mathcal{L}_{r}$ to $\mathcal{C}$ at $z=-\infty$ and $z=+\infty$, vanish. 
We are now ready to evaluate the wake potential $W_{z}^{(m=0)}$ by writing

$$
W_{z}^{(m=0)}(r, s)=-\frac{1}{Q} \int_{-\infty}^{+\infty}\left[\bar{e}_{z}^{(r)}\right]^{(m=0)}(r, z, s) d z=-\frac{1}{Q} \int_{\mathcal{L}_{r}} \overrightarrow{\mathrm{S}}^{(m=0)} \cdot d \vec{l}
$$

Equation (20) then implies that $W_{z}^{(m=0)}$ is $r$-independent and is given by

$$
W_{z}^{(m=0)}(s)=-\frac{1}{Q} \int_{\mathcal{C}}\left[\bar{e}_{z}^{(r)}(r, z, s) d z+\left(\bar{e}_{r}^{(r)}+c \bar{b}_{\theta}^{(r)}\right)(r, z, s) d r\right]^{(m=0)}
$$

Using Eqs.(5) and Eqs.(18), we can finally express the longitudinal potential $W_{z}^{(m=0)}$ in terms of the e.m. fields $(\mathbf{E}, \mathbf{B})^{(m=0)}$ in the structure, as follows

$$
\begin{gathered}
W_{z}^{(m=0)}(s)=-\frac{1}{Q} \int_{\mathcal{C}}\left[E_{z} d z+\left(E_{r}+c B_{\theta}\right) d r\right]^{(m=0)}(r, z, t(z, s)) \\
+\frac{1}{\pi \epsilon_{0}} \ln \left[\frac{r_{\text {out }}}{r_{\text {in }}}\right] \lambda(s)
\end{gathered}
$$

with $r_{\text {in }}$ and $r_{\text {out }}$ the end radii of the contour $\mathcal{C}$. For a contour ending on the beam tubes, like $\overline{\mathcal{C}}$ in Fig.1, the integration along the tubes vanishes since $E_{z}=0$ on a perfectly conducting boundary.

\subsubsection{THE $m \geq 1 \mathrm{CASE}$}

For $m \geq 1$, we make the assumption, which will be justified later, that the radii of the beam tubes are equal to $a$ on both sides of the structure. In order to deform the integration contour to the beam tubes, we need the asymptotic expression of the fields $(\mathbf{E}, \mathbf{B})^{(r)}$ for $z \rightarrow \pm \infty$. It is given by the fields in a perfectly conducting tube of radius $a$, which can be derived from the potentials

$$
\begin{gathered}
\phi(r, \theta, z, t)=\frac{Q}{2 \pi \epsilon_{0}} \lambda(c t-z)\left[\ln \left(\frac{a}{r_{>}}\right)+\sum_{m=1}^{\infty} \frac{\cos (m \theta)}{m}\left(\left(\frac{r_{<}}{r_{>}}\right)^{m}-\left(\frac{r r_{0}}{a^{2}}\right)^{m}\right)\right] \\
\mathbf{A}(r, \theta, z, t)=\hat{\mathbf{z}} \phi(r, \theta, z, t) / c
\end{gathered}
$$

with $r_{>}=\sup \left(r, r_{0}\right)$ and $r_{<}=\inf \left(r, r_{0}\right)$. Since the fields in free space are given by the same potentials with $a=+\infty$, the asymptotic form of the radiated fields $(\mathbf{E}, \mathbf{B})^{(r)}$ for $z \rightarrow \pm \infty$ is derived from the potentials

$$
\begin{gathered}
\phi^{(r)}(r, \theta, z, t)=-\frac{Q}{2 \pi \epsilon_{0}} \lambda(c t-z) \sum_{m=1}^{\infty} \frac{\cos (m \theta)}{m}\left(\frac{r r_{0}}{a^{2}}\right)^{m} \\
\mathbf{A}^{(r)}(r, \theta, z, t)=\hat{\mathbf{z}} \phi^{(r)}(r, \theta, z, t) / c
\end{gathered}
$$

Hence, one can easily obtain

$$
\begin{gathered}
\lim _{z \rightarrow \pm \infty} \bar{e}_{r}^{(r)}(r, z, s)=-\lim _{z \rightarrow \pm \infty} \bar{e}_{\theta}^{(r)}(r, z, s)=\lim _{z \rightarrow \pm \infty} c \bar{b}_{r}^{(r)}(r, z, s) \\
=\lim _{z \rightarrow \pm \infty} c \bar{b}_{\theta}^{(r)}(r, z, s)=\frac{Q}{2 \pi r \epsilon_{0}} \lambda(s) \sum_{m=1}^{\infty}\left(\frac{r r_{0}}{a^{2}}\right)^{m}
\end{gathered}
$$


and

$$
\lim _{z \rightarrow \pm \infty} \bar{e}_{z}^{(r)}(r, z, s)=\lim _{z \rightarrow \pm \infty} c \bar{b}_{z}^{(r)}(r, z, s)=0
$$

From these limiting behaviors one can deduce the following identities

$$
\int_{\mathcal{L}_{r}} \overrightarrow{\mathrm{S}}^{(m)}(r, z, s) \cdot d \vec{l}=\int_{\mathcal{C}} \overrightarrow{\mathrm{S}}^{(m)}\left(r^{\prime}, z, s\right) \cdot d \vec{l}+\frac{Q}{\pi \epsilon_{0}} \lambda(s) \frac{r_{0}^{m}}{m a^{2 m}}\left(r_{\text {in }}^{2 m}-r_{\text {out }}^{2 m}\right)
$$

and

$$
\int_{\mathcal{L}_{r}} \overrightarrow{\mathrm{D}}^{(m)}(r, z, s) \cdot d \vec{l}=\int_{\mathcal{C}} \overrightarrow{\mathrm{D}}^{(m)}\left(r^{\prime}, z, s\right) \cdot d \vec{l}
$$

with $r_{\text {in }}$ and $r_{\text {out }}$ defined in Fig.1. Applying Eq.(28) in the case where the contour $\mathcal{C}$ is the axis itself $\mathcal{L}_{0}$, leads to

$$
\int_{\mathcal{L}_{r}} \overrightarrow{\mathrm{S}}^{(m)}(r, z, s) \cdot d \vec{l}=\int_{\mathcal{L}_{0}} \overrightarrow{\mathrm{S}}^{(m)}(r=0, z, s) \cdot d \vec{l}=0
$$

and thus

$$
\int_{\mathcal{C}} \overrightarrow{\mathrm{S}}^{(m)}\left(r^{\prime}, z, s\right) \cdot d \vec{l}=\frac{Q}{\pi \epsilon_{0}} \lambda(s) \frac{r_{0}^{m}}{m a^{2 m}}\left(r_{\text {out }}^{2 m}-r_{\text {in }}^{2 m}\right)
$$

Another consequence of Eq.(30) is the identity

$$
\int_{-\infty}^{+\infty}\left[\bar{e}_{z}^{(r)}(r, z, s)\right]^{(m)} d z=-\int_{-\infty}^{+\infty}\left[c \bar{b}_{z}^{(r)}(r, z, s)\right]^{(m)} d z
$$

We can now calculate the wake potential $W_{z}^{(m)}$, given by

$$
\begin{aligned}
W_{z}^{(m)}(r, \theta, s) & =-\frac{1}{Q} \int_{-\infty}^{+\infty} E_{z}^{(m)}(r, \theta, z, t(z, s)) d z \\
& =-\frac{\cos (m \theta)}{Q} \int_{-\infty}^{+\infty}\left[\bar{e}_{z}^{(r)}\right]^{(m)}(r, z, s) d z
\end{aligned}
$$

Using Eq.(32) and then Eq.(29), one can write

$$
\begin{aligned}
W_{z}^{(m)}(r, \theta, s) & =-\frac{\cos (m \theta)}{2 Q} \int_{-\infty}^{+\infty}\left[\bar{e}_{z}^{(r)}-c \bar{b}_{z}^{(r)}\right]^{(m)}(r, z, s) d z \\
& =-\frac{\cos (m \theta)}{2 Q} r^{m} \int_{\mathcal{L}_{r}} \overrightarrow{\mathrm{D}}^{(m)}(r, z, s) \cdot d \vec{l} \\
& =-\frac{\cos (m \theta)}{2 Q} r^{m} \int_{\mathcal{C}} \overrightarrow{\mathrm{D}}^{(m)}\left(r^{\prime}, z, s\right) \cdot d \vec{l}
\end{aligned}
$$

In the last equation, we get the explicit $(r, \theta)$-dependence of $W_{z}^{(m)}$ since the integral does not depend on the contour $\mathcal{C}$. However, even if the contour ends on the beam tubes, like $\overline{\mathcal{C}}$ in Fig.1, the integral extends to $+\infty$ and $-\infty$ because the component $\bar{b}_{z}^{(r)}$ does not vanish 
on the metallic boundary. It is possible to eliminate the integral over the beam tubes by using Eq.(31) for the contour $\overline{\mathcal{C}}$, as follows

$$
\begin{aligned}
W_{z}^{(m)}(r, \theta, s) & =-\frac{\cos (m \theta)}{2 Q} r^{m}\left(\int_{\overline{\mathcal{C}}} \overrightarrow{\mathrm{D}}^{(m)}\left(r^{\prime}, z, s\right) \cdot d \vec{l}+\frac{1}{a^{2 m}} \int_{\overline{\mathcal{C}}} \overrightarrow{\mathrm{S}}^{(m)}\left(r^{\prime}, z, s\right) \cdot d \vec{l}\right) \\
& =-\frac{\cos (m \theta)}{2 Q} \frac{r^{m}}{a^{m}} \int_{\overline{\mathcal{C}}} d z\left[\bar{e}_{z}^{(r)}\left(\frac{a^{m}}{r^{\prime m}}+\frac{r^{\prime m}}{a^{m}}\right)-c \bar{b}_{z}^{(r)}\left(\frac{a^{m}}{r^{\prime m}}-\frac{r^{\prime m}}{a^{m}}\right)\right]^{(m)} \\
& +d r^{\prime}\left[\left(\bar{e}_{r}^{(r)}+c \bar{b}_{\theta}^{(r)}\right)\left(\frac{a^{m}}{r^{\prime m}}+\frac{r^{\prime m}}{a^{m}}\right)+\left(\bar{e}_{\theta}^{(r)}-c \bar{b}_{r}^{(r)}\right)\left(\frac{a^{m}}{r^{\prime m}}-\frac{r^{\prime m}}{a^{m}}\right)\right]^{(m)}
\end{aligned}
$$

where it is understood that the argument of the fields is $\left(r^{\prime}, z, s\right)$. Using Eq.(5) to express the radiated fields in terms of the real fields in the structure and the fields in free space, one gets

$$
\begin{aligned}
W_{z}^{(m)}(r, \theta, s) & =-\frac{\cos (m \theta)}{2 Q} \frac{r^{m}}{a^{m}} \int_{\bar{c}} d z\left[e_{z}\left(\frac{a^{m}}{r^{\prime m}}+\frac{r^{\prime m}}{a^{m}}\right)-c b_{z}\left(\frac{a^{m}}{r^{\prime m}}-\frac{r^{\prime m}}{a^{m}}\right)\right]^{(m)} \\
& +d r^{\prime}\left[\left(e_{r}+c b_{\theta}\right)\left(\frac{a^{m}}{r^{\prime m}}+\frac{r^{\prime m}}{a^{m}}\right)+\left(e_{\theta}-c b_{r}\right)\left(\frac{a^{m}}{r^{\prime m}}-\frac{r^{\prime m}}{a^{m}}\right)\right]^{(m)}
\end{aligned}
$$

where the argument of the fields is now $\left(r^{\prime}, z, t(z, s)\right)$. Notice that the fields in free space $\mathbf{E}^{(0)}$ and $\mathbf{B}^{(0)}$ do not contribute to this result since their longitudinal components are zero and their transverse components depend only on $r^{\prime}$ and $s$. Therefore, their integral along $\overline{\mathcal{C}}$ amounts to an integral over $r^{\prime}$ from $a$ to $a$, and vanishes. In Eq.(36) the wake field integration along the beam tubes drops out since $e_{z}=0$ and $r^{\prime}=a$ on the metallic boundary. In the case of unequal beam tube radii, only one of these integrals can be eliminated in this way. Then the method is not much better than the direct wake field integration along $\mathcal{L}_{r}$. It can be improved, however, by placing an additional step at some distance in one of the beam tubes, and subtracting the contribution of this step to the wake potentials.

\subsection{The transverse potential}

The transverse potential can be derived from the longitudinal one by applying the PanofskyWenzel theorem

$$
\partial_{s} \mathbf{W}_{\perp}(r, \theta, s)=\nabla_{\perp} W_{z}(r, \theta, s)
$$

Using the expression of $W_{z}$ from Eq.(35) and the homogeneous Maxwell equations fulfilled by the fields $\left(\mathbf{E}^{(r)}, \mathbf{B}^{(r)}\right)$, it is easy to show that the solution of Eq.(37) is given by [6]

$$
\mathbf{W}_{\perp}(r, \theta, s)=\sum_{m=1}^{\infty} r^{m-1}(\cos (m \theta) \hat{\mathbf{r}}-\sin (m \theta) \hat{\boldsymbol{\theta}}) w^{(m)}(s)
$$


with

$$
\begin{aligned}
w^{(m)}(s) & =\frac{1}{2 Q a^{m}} \int_{\bar{c}} d z\left[r^{\prime}\left(\bar{e}_{r}^{(r)}-c \bar{b}_{\theta}^{(r)}\right)\left(\frac{a^{m}}{r^{\prime m}}-\frac{r^{\prime m}}{a^{m}}\right)-r^{\prime}\left(\bar{e}_{\theta}^{(r)}+c \bar{b}_{r}^{(r)}\right)\left(\frac{a^{m}}{r^{\prime m}}+\frac{r^{\prime m}}{a^{m}}\right)\right]^{(m)} \\
& -d r^{\prime}\left[r^{\prime} \bar{e}_{z}^{(r)}\left(\frac{a^{m}}{r^{\prime m}}-\frac{r^{\prime m}}{a^{m}}\right)-r^{\prime} c \bar{b}_{z}^{(r)}\left(\frac{a^{m}}{r^{\prime m}}+\frac{r^{\prime m}}{a^{m}}\right)\right]^{(m)}
\end{aligned}
$$

In terms of e.m. fields $(\mathbf{E}, \mathbf{B})$ in the structure, this gives

$$
\begin{aligned}
w^{(m)}(s) & =\frac{1}{2 Q a^{m}} \int_{\overline{\mathcal{C}}} d z\left[r^{\prime}\left(e_{r}-c b_{\theta}\right)\left(\frac{a^{m}}{r^{\prime m}}-\frac{r^{\prime m}}{a^{m}}\right)-r^{\prime}\left(e_{\theta}+c b_{r}\right)\left(\frac{a^{m}}{r^{\prime m}}+\frac{r^{\prime m}}{a^{m}}\right)\right]^{(m)} \\
& -d r^{\prime}\left[e_{z}\left(\frac{a^{m}}{r^{\prime m}}-\frac{r^{\prime m}}{a^{m}}\right)-c b_{z}\left(\frac{a^{m}}{r^{\prime m}}+\frac{r^{\prime m}}{a^{m}}\right)\right]^{(m)}
\end{aligned}
$$

where the argument of the fields is $\left(r^{\prime}, z, t(z, s)\right)$. Again, the fields in free space $\mathbf{E}^{(0)}$ and $\mathbf{B}^{(0)}$ do not contribute to this result since their longitudinal components and the combination of their transverse components appearing in Eq.(40), are zero.

\section{Computer Implementation}

\subsection{The Integration Contour}

For a general path of integration, the wake potentials are given as integrals over combinations of the electric and magnetic field strengths, depending on the angle of the integration path. For the particular case of contours parallel to the $r$ and $z$ axis, the integration is considerably simplified. For this reason, the program ABCI has an option which uses a path of integration that starts as usual along the beam tube, then descends radially to pass underneath the smallest material structure radius. It then rises again to the radius of the exit tube and moves along it to the end of the structure (Fig.2).

A proper integration path is actually chosen automatically as soon as some material point has a radius smaller than the beam tube. Alternatively, the three coordinates $\mathrm{ZCF}, \mathrm{ZCT}$, and RWAK (see Fig.2) can also be chosen as input parameters. In particular for structures with a complicated boundary extending to the inside of the beam tubes, such as disk-loaded waveguides with many cells, this technique leads to a considerable saving in computing time.

The new version of ABCI has a "moving mesh", which not only limits the field computations to a small region around the bunch (as in the old "WINDOW" option), but actually needs to store only these mesh points at any given moment. This reduces the required computer memory considerably, and very fine meshes can be used. Furthermore, in the newer versions of $\mathrm{ABCI}$, the radial mesh size can be varied over the structure, permitting to use a fine mesh only where actually needed. With these improvements, the program allows computation of wake fields for structures far too complicated for older codes. 


\subsection{Examples}

Here we show a number of structures which have been evaluated using the latest version (6.2.4) of the program ABCI. We first consider a collimator, i.e. a simple constriction of a beam tube (Fig.3). The wake potentials are computed easily with this program by using the metallic boundary as contour of integration. Hence the results do not depend on the length of the beam tubes, where the integral is zero, provided it is significantly larger than the bunch length. The loss factors thus obtained agree with the result of integrating along a straight line at the inner radius of the collimator with asymptotically long beam tubes and after subtraction of the wake of a tube alone ("numerical noise correction", similar to the 'WAKCOR' integration in TBCI[7]), but are found easier and faster. This is shown in Fig.4 where the longitudinal loss factor of a bunch with $\sigma_{z}=0.5 \mathrm{~mm}$ is plotted as function of the beam tube length using the old method and seen to converge to the value obtained directly with ABCI.

Next we show computations of the wake potentials and loss factors of tubes with a step to a smaller radius ("step-in") (Figs.5,6) and to a larger one ("step out") (Figs.7,8). The sum of the loss factors equals exactly that of a pillbox cavity (Fig.9), if the two steps are sufficiently separated (Fig.10). It is thus possible to split the computation of long geometries in small parts and thus save memory and cpu time.

Another example is the wake of a 20 -cell "constant gradient" accelerating structure, where the inner and outer radii of each cell are different. By choosing the radial step size small only where required (near the irises and rear walls), and thanks to the "moving mesh" option, the wakes and loss factors of very short bunches can be computed in a reasonable time. The number of mesh points covering the whole structure with a uniform mesh of the same resolution would be $10^{7}$ to $10^{8}$, and thus could not be handled even with fastest computers existing today.

When the wake is desired far behind the bunch, the size of the window and hence the computing time increases, and thus is presently still limited to a fraction of the structure length. In the example given, 1400 seconds of cpu-time on the CERN-IBM were needed to obtain the wake up to $6 \mathrm{~mm}$ behind a Gaussian bunch with an RMS of $0.17 \mathrm{~mm}$, corresponding to a distance of 36 sigma.

As a final example we give the computation of the transverse wakes in a 30 -cell staggertuned disk loaded waveguide (Figs.11,12) for a Linear Collider. It has been computed for a distance of up to several centimeters behind the bunch, which needs a cpu-time of an hour or more on the CERN-IBM. Longer distances could be obtained by running the program on a dedicated workstation, which will be installed in the near future.

\section{Conclusion}

In practical calculations of wake potentials created by axisymmetric cavities, one usually evaluates them by integrating along the cavity gap at the beam tube radius. We have generalized this method by showing that the wake potentials, of any multipole order, are given by integrals over the wake fields along any arbitrary contour spanning the structure longitudinally. By so doing we have extended the range of applications to structures of more complicated shape. The integration of wake fields along a well chosen contour permits a large 
savings in computer capacity. In particular, the integration along a structure extending to the inside of the beam tubes - such as a collimator or iris - has become much easier with this method. Also the $m=0$ wake potential of structures with unequal beam tubes can be found in this manner. The new method of integration has been implemented in the code ABCI (versions 4 or higher) which can choose the proper contour automatically or as selected by the user.

\section{References}

[1] T. Weiland, NIM 216 (1983), 31

[2] R. L. Gluckstern and F. Neri, IEEE NS-32, 52403 (1985)

[3] O. Napoly, Part. Acc., 3615 (1991)

[4] Y. H. Chin, Report LBL-33092 and CERN-SL/92-49 (1992)

[5] W. Panofsky and W. Wenzel, Rev. Sci. Instrument, 27967 (1956)

[6] This solution has been derived by M. Elabed, unpublished

[7] T. Weiland, Report DESY 82-015 (1982) 


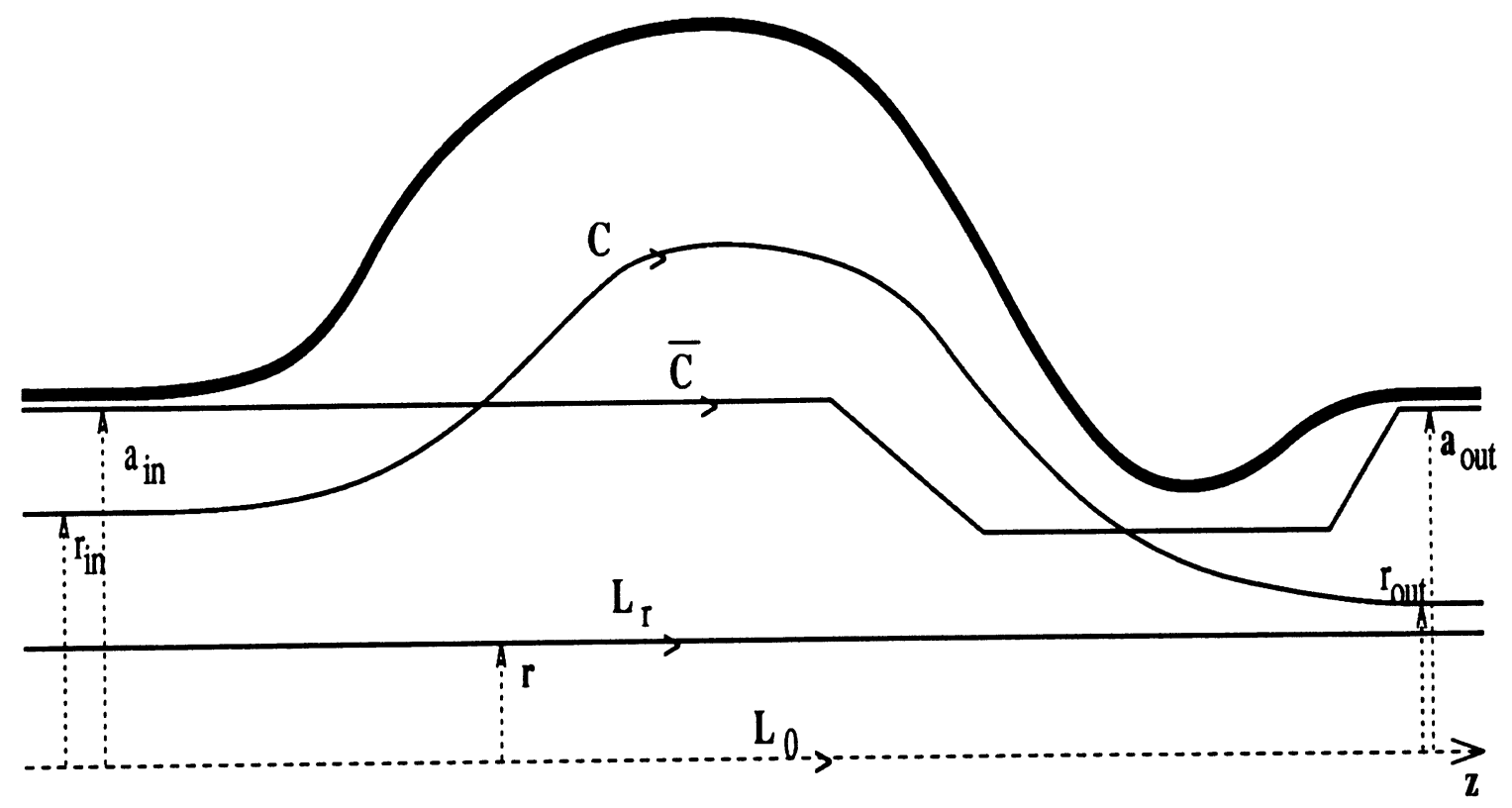

Figure 1: General Cavity and Integration Contours

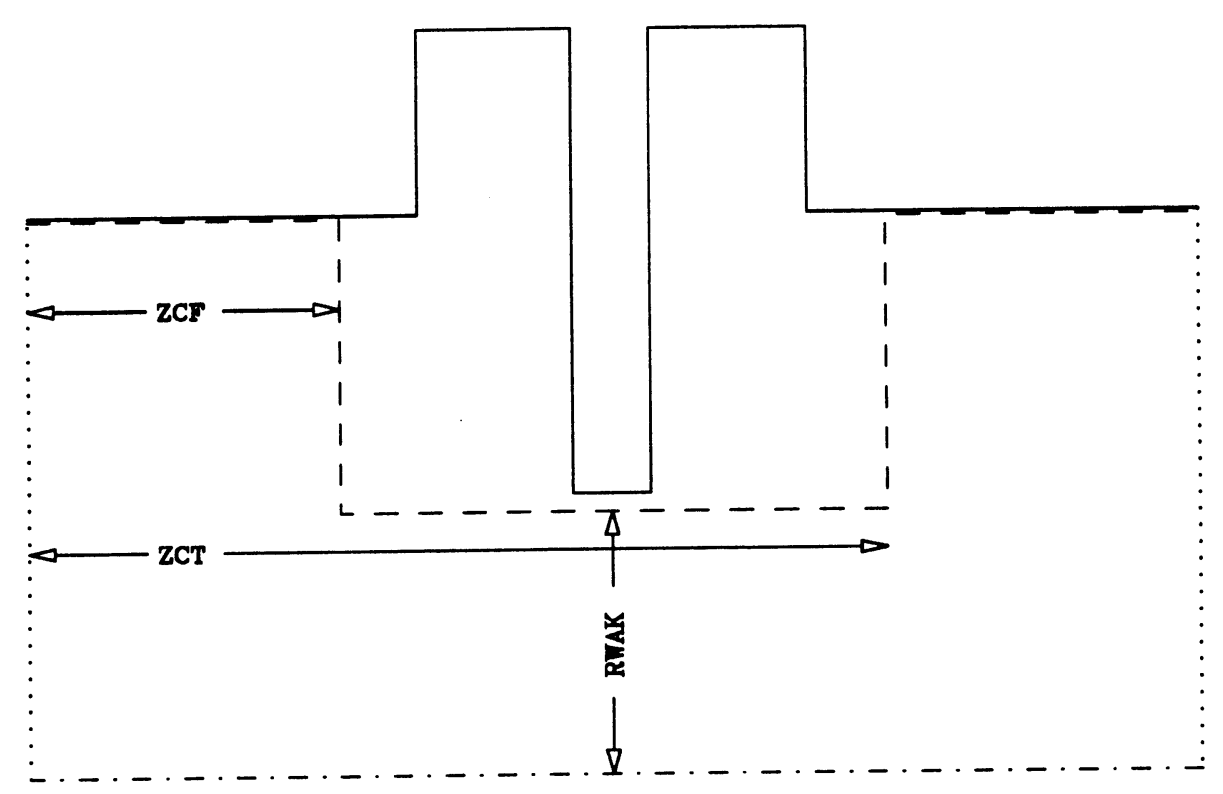

Figure 2: Contour Parameters in Program ABCI 


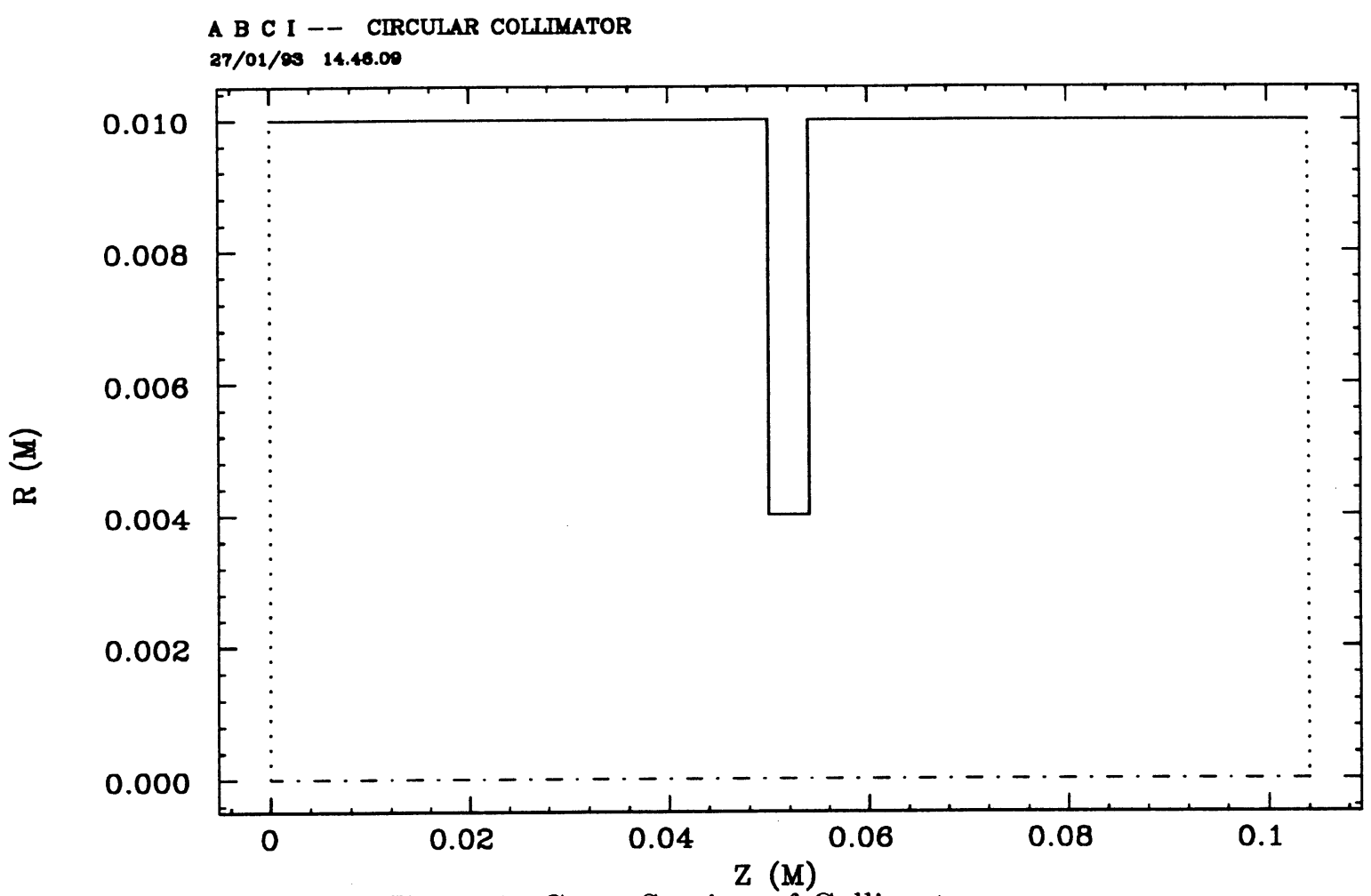

Figure 3: Cross Section of Collimator

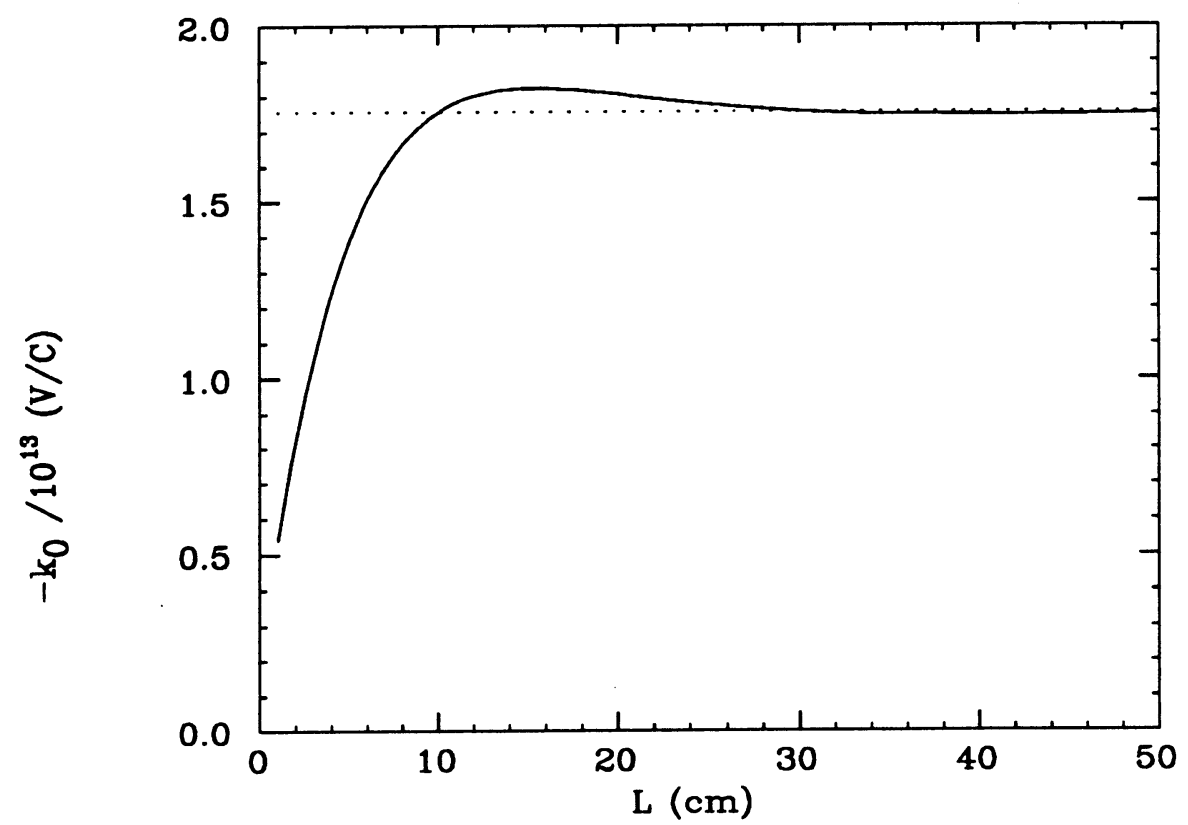

Figure 4: Longitudinal Loss Factor of Collimator vs. Beam Tube Length 


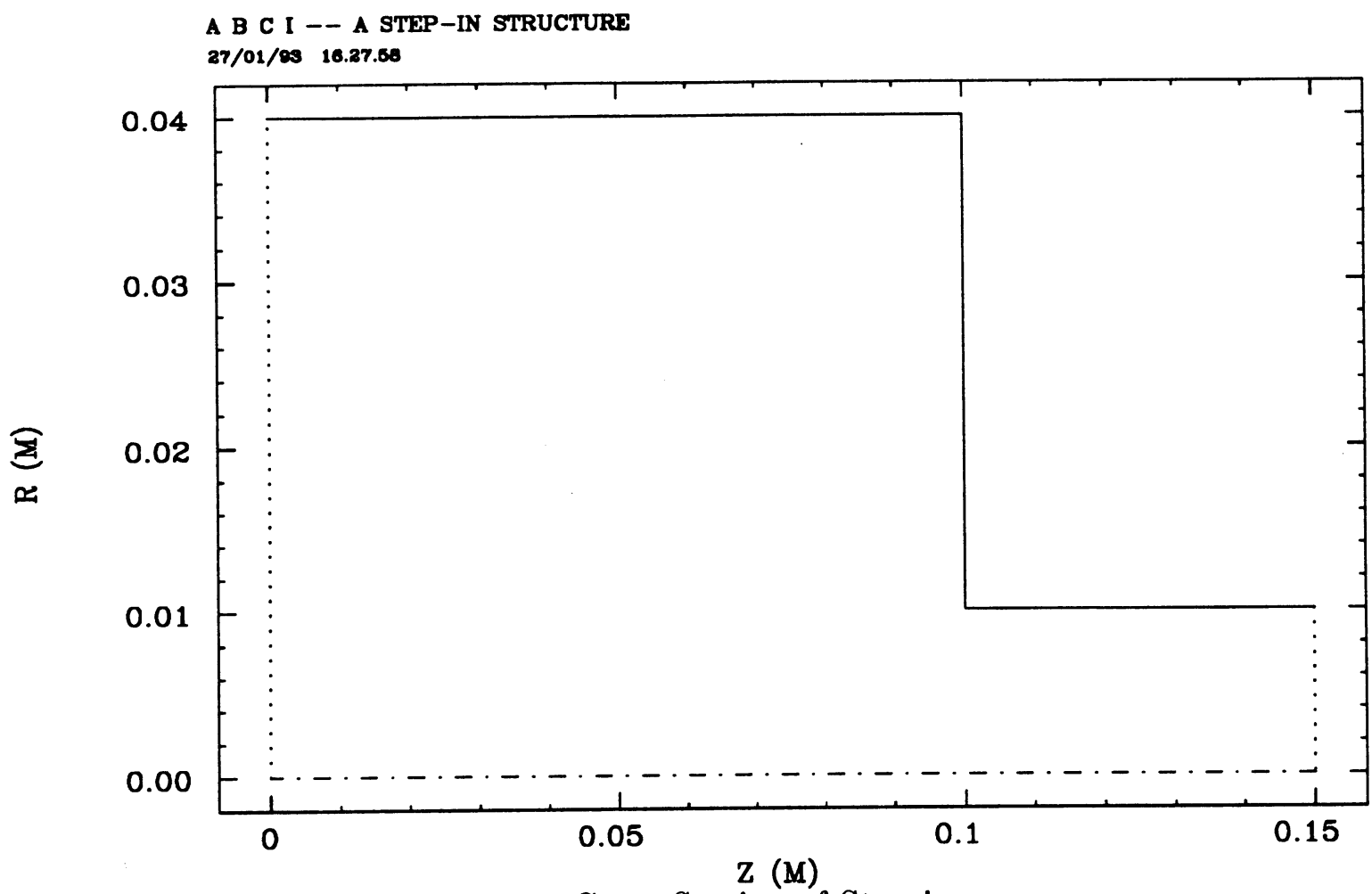

Figure 5: Cross Section of Step-in

- WAKE POTENTIALS -

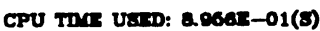

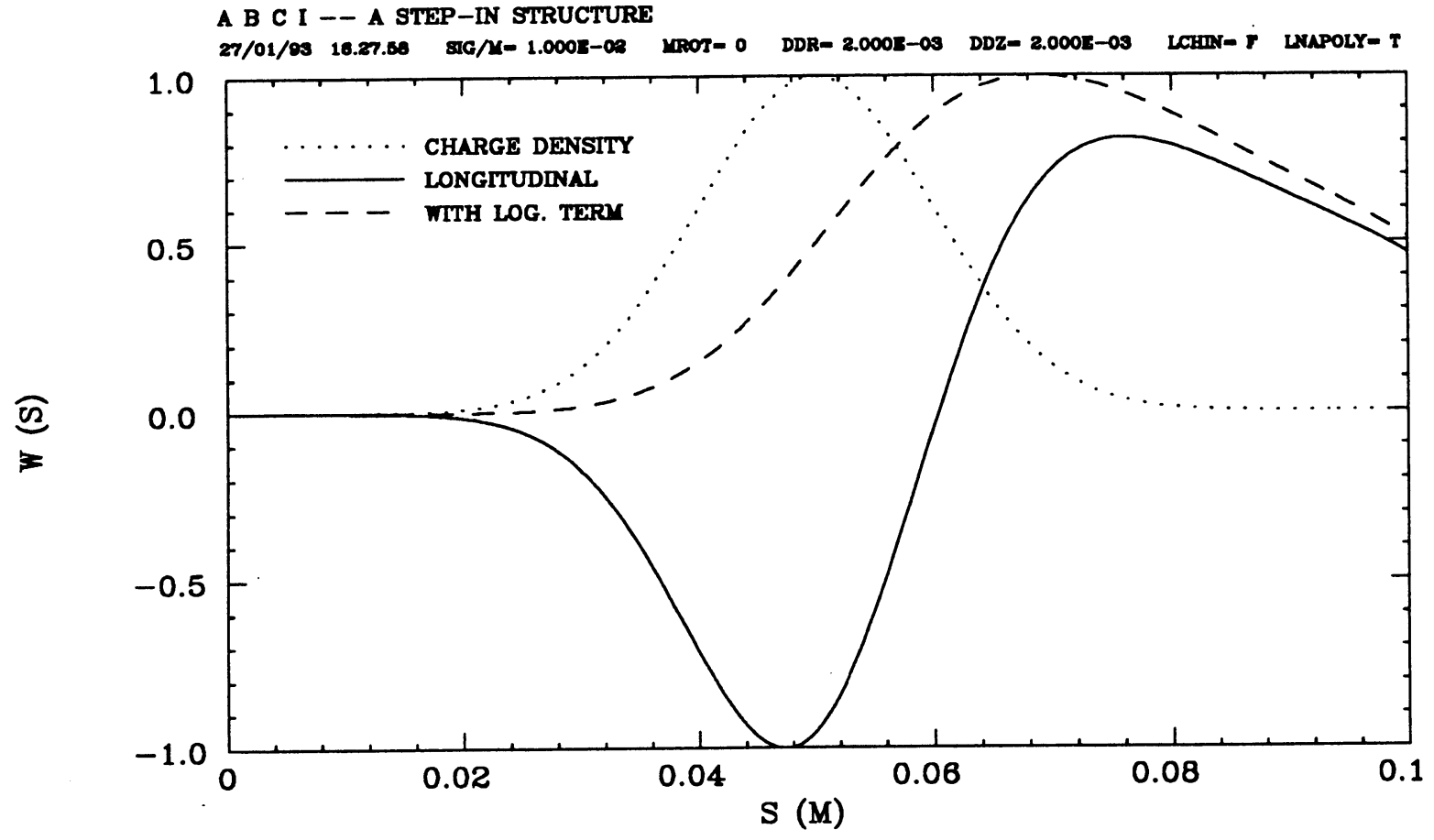

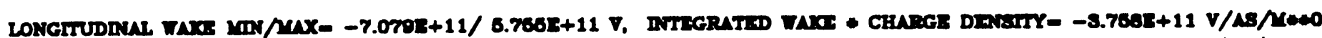

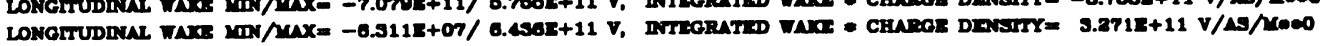

Figure 6: Wake Potential of step-in 


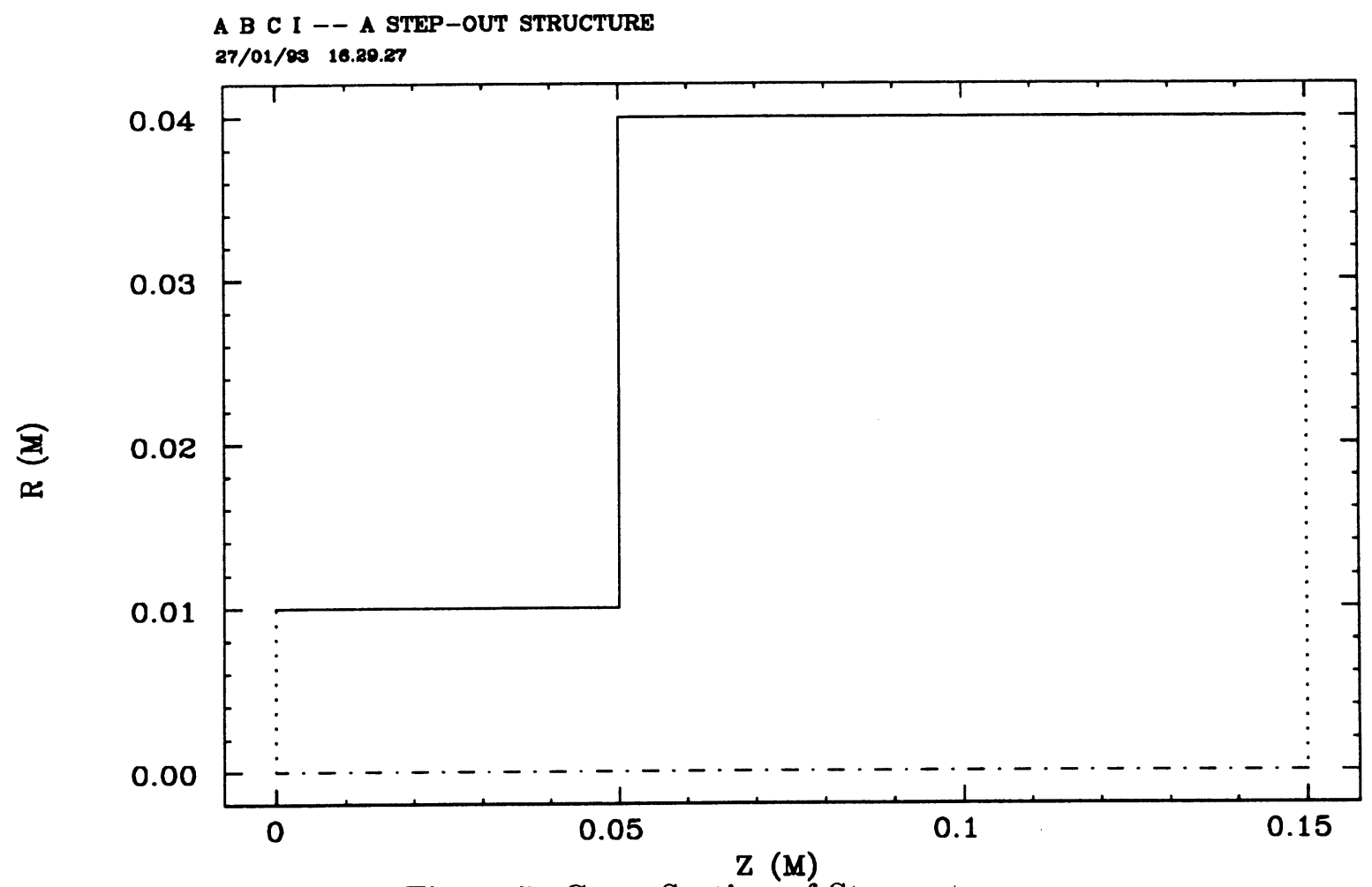

Figure 7: Cross Section of Step-out

- WAKE POTENTIALS -

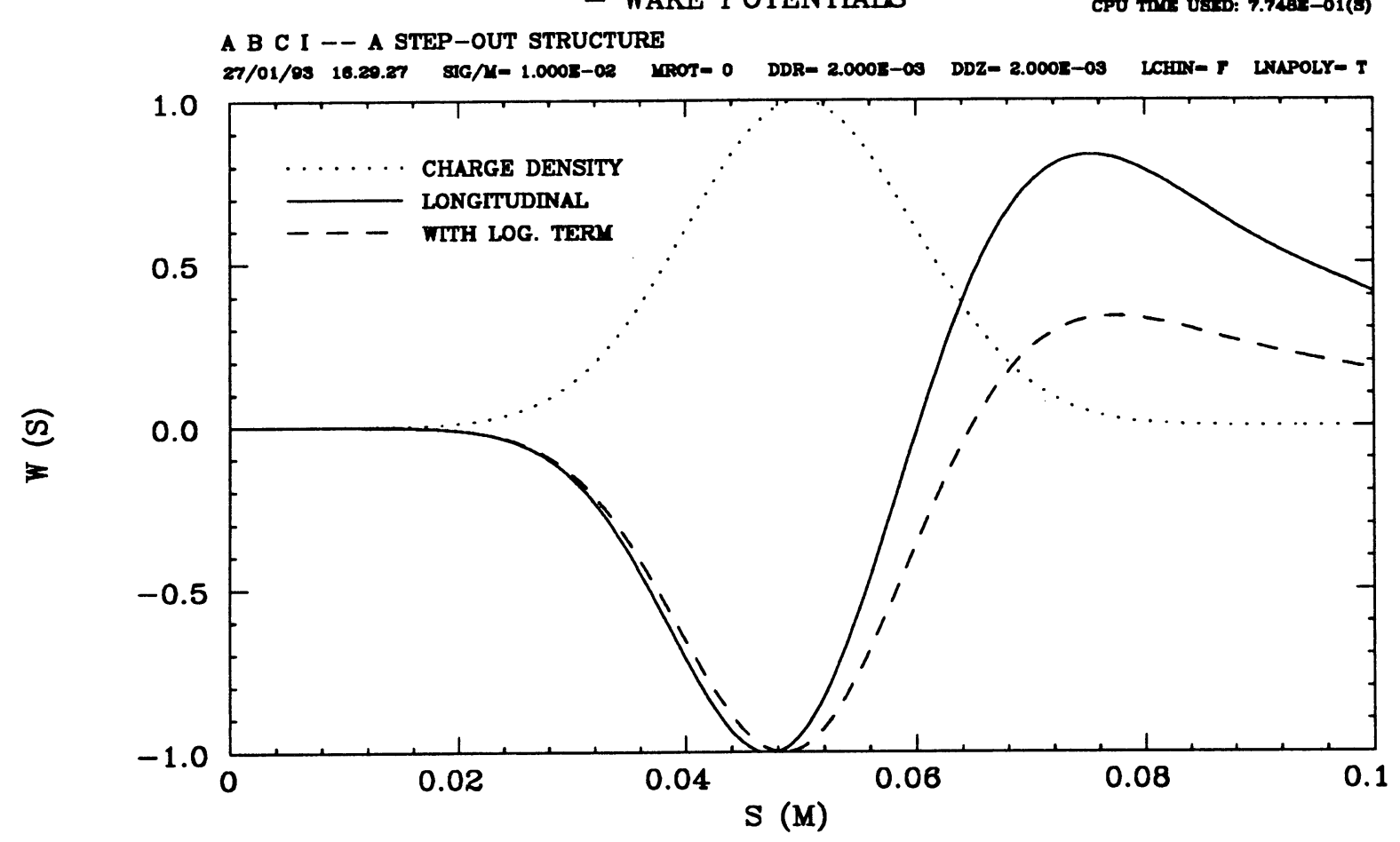

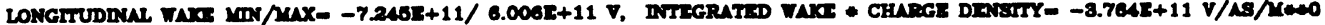

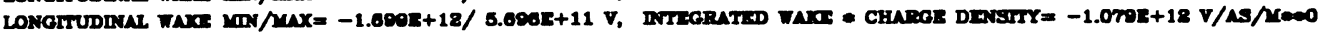

Figure 8: Wake Potential of step-out 


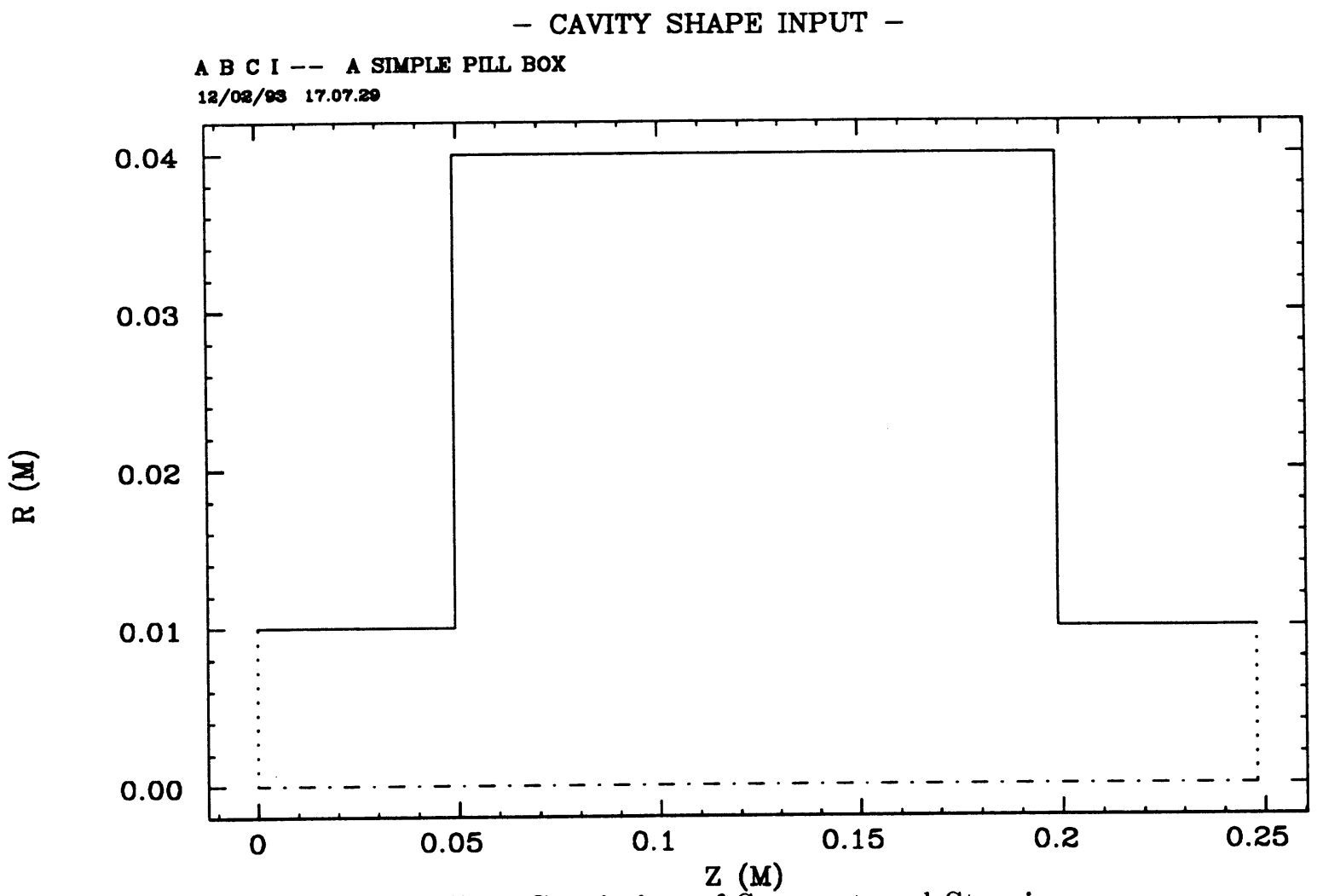

Figure 9: Pillbox Consisting of Step-out and Step-in

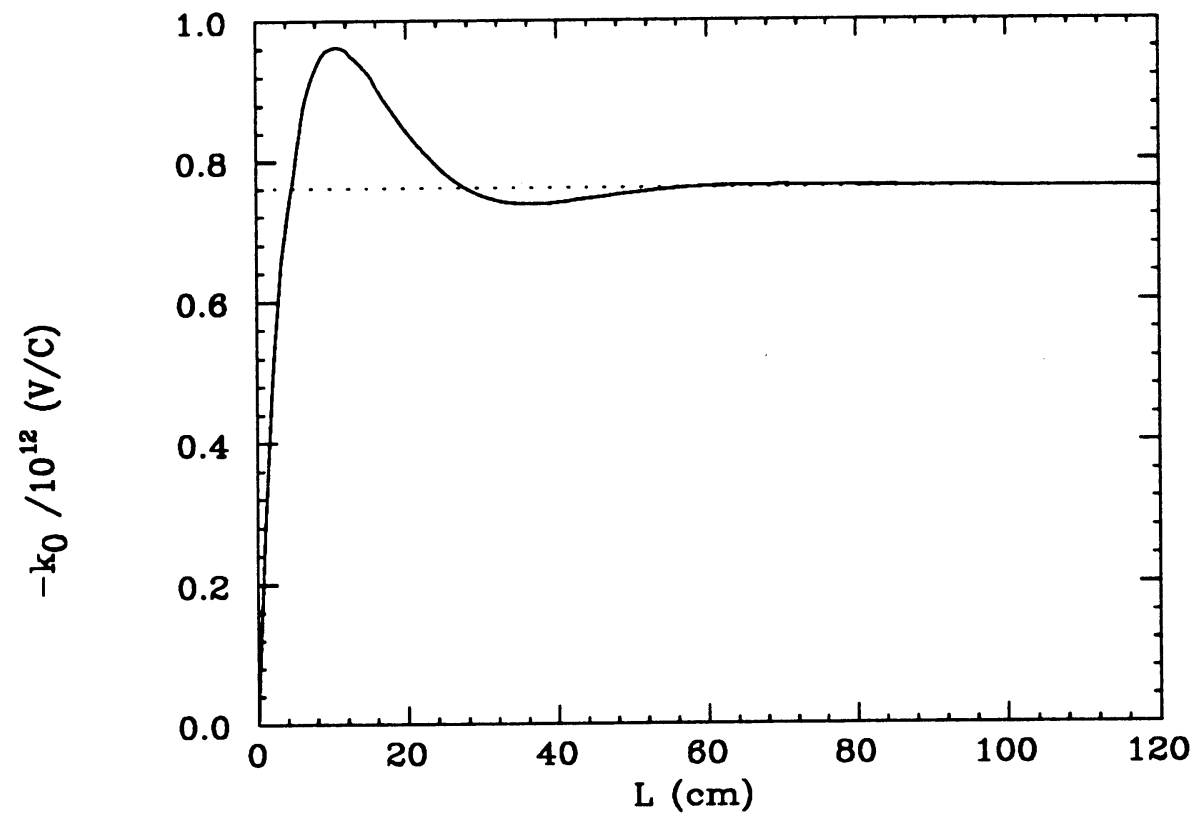

Figure 10: Sum of Loss Factors as Function of Distance Between Steps 
- CAVITY SHAPE INPUT -

A B C I -- CLIC STRUCTURE 20 CEIL (CONSTANT GRADIENT)

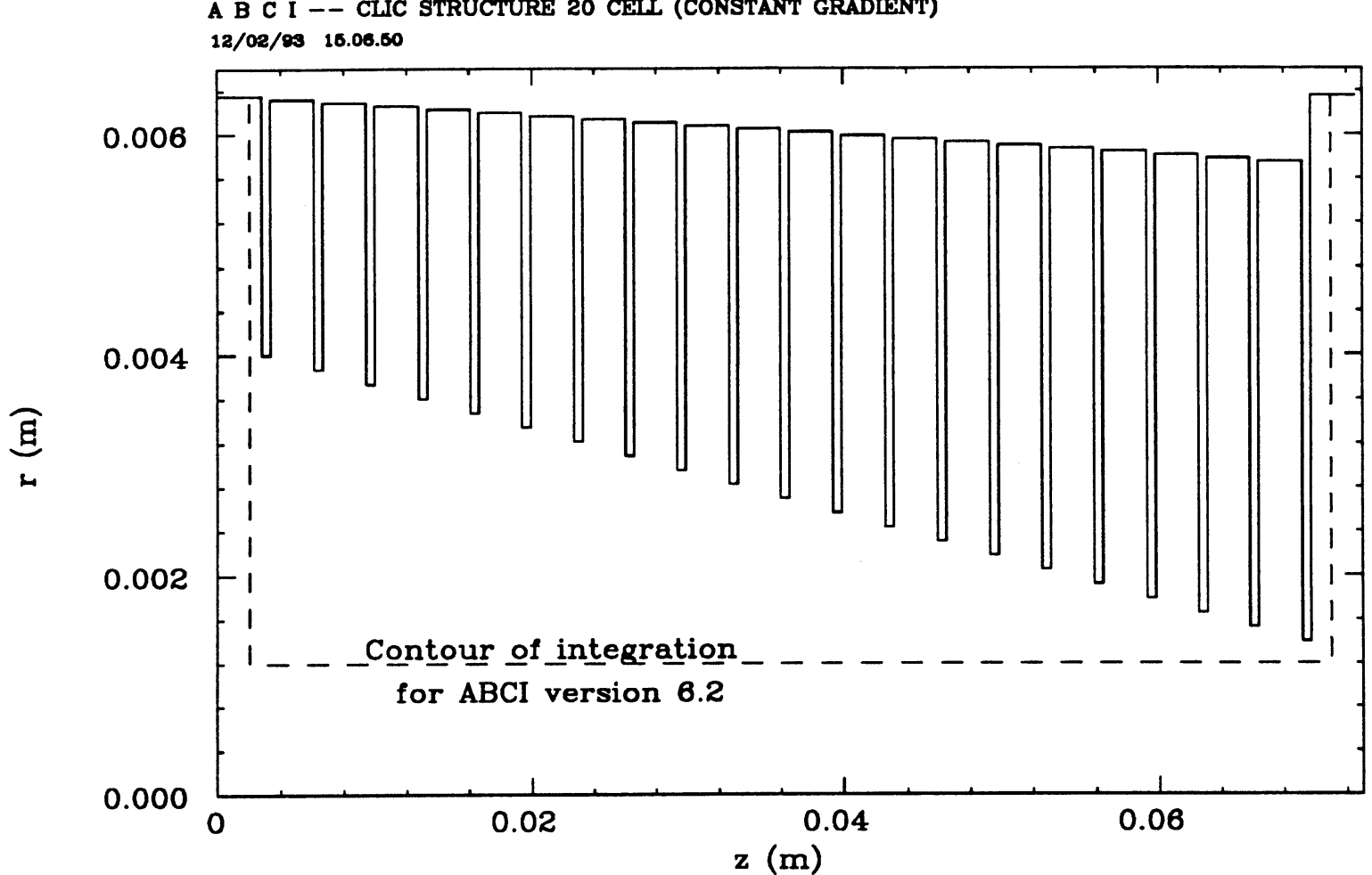

Figure 11: Constant Gradient Structure for CERN Linear Collider

$$
\text { - WAKE POTENTIALS - }
$$

CPU TnE USTE: 1.401E+08(8)

A B C I - - CLIC. STRUCTURE 20 CELL (CONSTANT GRADIFNT)

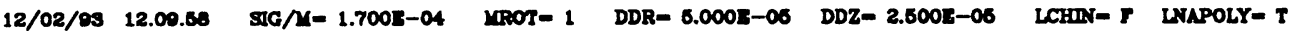

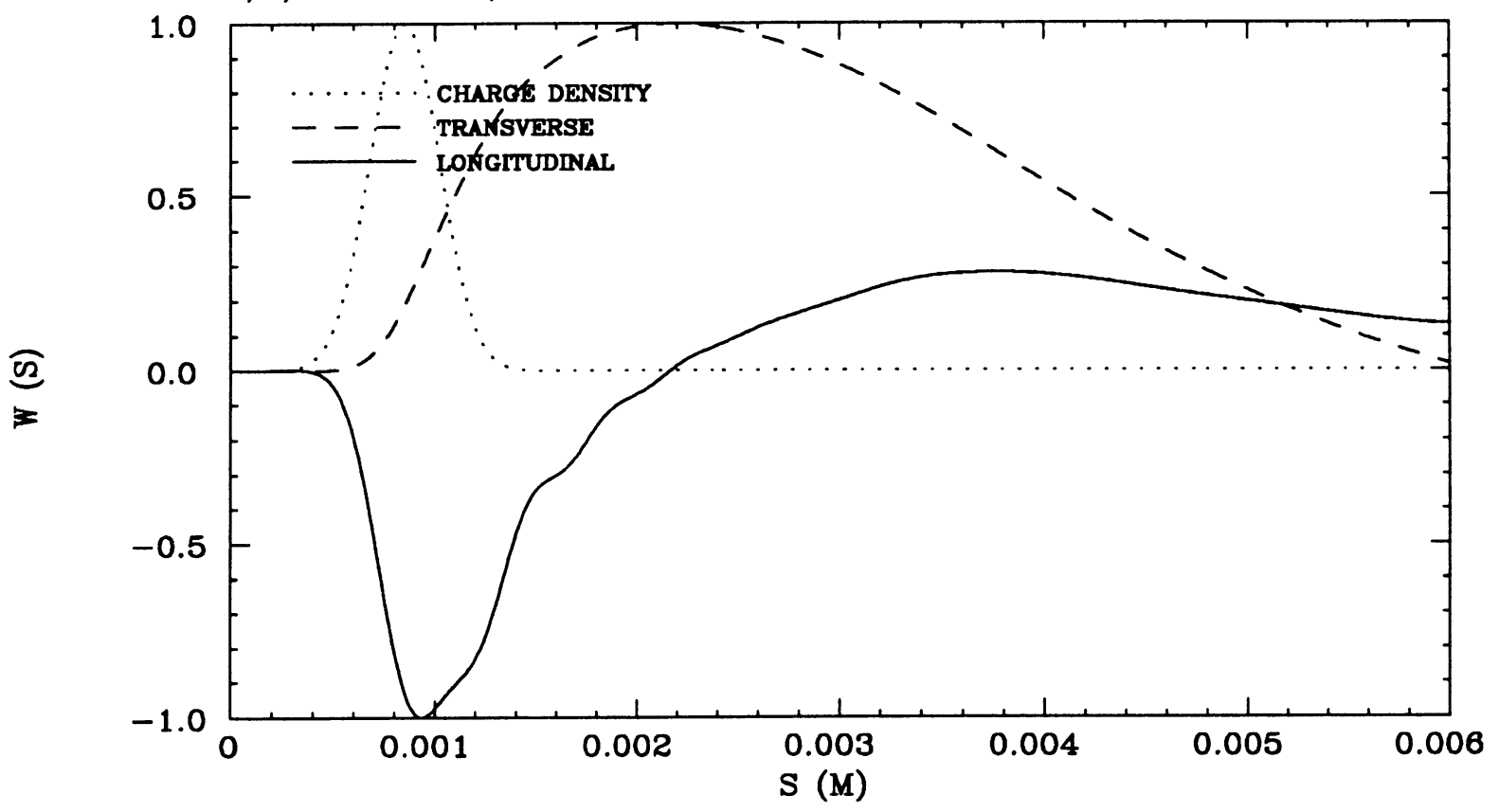

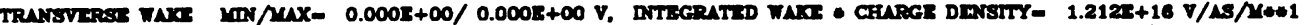

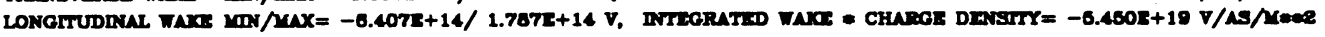

Figure 12: Transverse Wake of Constant Gradient Structure 